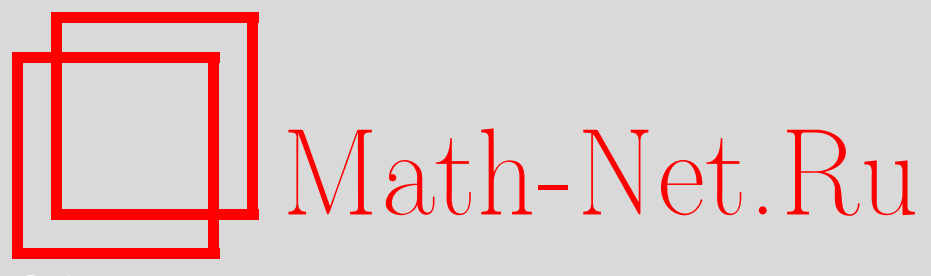

А. А. Муравлёв, О моментах остановки, связанных с падением и ростом броуновского движения со сносом, УМН, 2008, том 63, выпуск 6, 171-172

DOI: https://doi.org/10.4213/rm9257

Использование Общероссийского математического портала Math-Net.Ru подразумевает, что вы прочитали и согласны с пользовательским соглашением http://www . mathnet.ru/rus/agreement

Параметры загрузки:

IP : 54.84 .234 .179

26 апреля 2023 г., 12:23:59

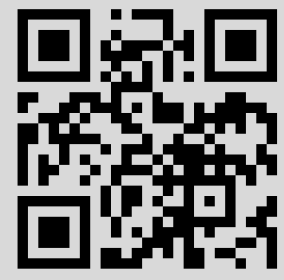




\section{О моментах остановки, связанных с падением и ростом броуновского движения со сносом}

\section{А. А. Муравлёв}

1. Пусть $B^{\mu}=\left(B_{t}^{\mu}\right)_{t \geqslant 0}-$ броуновское движение с локальным сносом $\mu \in \mathbb{R}$, т. е. $B_{t}^{\mu}=B_{t}+\mu t$, где $B=\left(B_{t}\right)_{t \geqslant 0}-$ стандартное броуновское движение. Для фиксированного $\mu \in \mathbb{R}$ и $a>0, b>0$ введем моменты:

$$
\begin{gathered}
\tau_{a}=\inf \left\{t \geqslant 0: \sup _{s \leqslant t} B_{s}^{\mu}-B_{t}^{\mu}=a\right\}, \\
\sigma_{b}=\inf \left\{t \geqslant 0: B_{t}^{\mu}-\inf _{s \leqslant t} B_{s}^{\mu}=b\right\}, \\
\gamma_{a b}=\tau_{a} \wedge \sigma_{b} .
\end{gathered}
$$

В случае $\mu=0$ будем обозначать эти моменты $\tau_{a}^{0}, \sigma_{b}^{0}$ и $\gamma_{a b}^{0}$. Положим

$$
\Delta=\sqrt{\mu^{2}+2 \lambda}, \quad \nu=\mu+\beta, \quad m_{a}=\frac{e^{2 \mu a}-2 \mu a-1}{e^{2 \mu a}+e^{-2 \mu a}-2}, \quad \lambda_{a}=\frac{e^{2 \mu a}-1}{2 \mu}
$$

и будем считать далее, что $\lambda \geqslant 0$. Вопрос о свойствах $\tau_{a}$ и $\sigma_{b}$ рассматривался во многих работах. Так, в [1] показано, что для момента $\tau_{a}$

$$
\begin{aligned}
& \mathbf{E} e^{-\lambda \tau_{a}}=\frac{e^{-\mu a}}{\operatorname{ch}(\Delta a)-(\mu / \Delta) \operatorname{sh}(\Delta a)}, \quad \mu \neq 0, \quad \mathbf{E} e^{-\lambda \tau_{a}^{0}}=\frac{1}{\operatorname{ch}(a \sqrt{2 \lambda})}, \\
& \mathbf{E} \tau_{a}=\frac{e^{2 \mu a}-2 \mu a-1}{2 \mu^{2}}, \quad \mu \neq 0, \quad \mathbf{E} \tau_{a}^{0}=a^{2} .
\end{aligned}
$$

Будем рассматривать случай $b \geqslant a>0$, так как при $a \geqslant b>0$ во всех формулах следует просто сделать замену $a \leftrightarrow b, \mu \rightarrow-\mu$ и $\nu \rightarrow-\nu$.

Основные результаты настоящей статьи состоят в следующем.

Теорема 1. Пусть $B_{t}^{\mu}-$ броуновское движение со сносом $\mu$, моменты $\tau_{a}, \sigma_{b} u \gamma_{a b}$ соответствуют $B_{t}^{\mu}$. Тогда:

1. $\quad \mathbf{E} e^{-\lambda \gamma_{a b}+\beta B_{\gamma_{a b}}^{\mu}}=\frac{e^{-\nu a}\left(1-e^{\nu(b-a)} e^{-\Delta(b-a) \operatorname{cth}(\Delta a)}\right)}{\operatorname{ch}(\Delta a)-(\nu / \Delta) \operatorname{sh}(\Delta a)}$

$$
+2 \frac{\operatorname{ch}(\Delta a) \operatorname{ch}(\nu a)-(\nu / \Delta) \operatorname{sh}(\Delta a) \operatorname{sh}(\nu a)-1}{\operatorname{sh}^{2}(\Delta a)\left(1-(\nu / \Delta)^{2}\right)} e^{\nu(b-a)} e^{-\Delta(b-a) \operatorname{cth}(\Delta a),}
$$

если $|\nu|<\Delta \operatorname{cth}(\Delta a)$

2. $\quad \mathbf{E} e^{-\lambda \gamma_{a b}}=\frac{e^{-\mu a}\left(1-e^{\mu(b-a)} e^{-\Delta(b-a) \operatorname{cth}(\Delta a)}\right)}{\operatorname{ch}(\Delta a)-(\mu / \Delta) \operatorname{sh}(\Delta a)}$

$+2 \frac{\operatorname{ch}(\Delta a) \operatorname{ch}(\mu a)-(\mu / \Delta) \operatorname{sh}(\Delta a) \operatorname{sh}(\mu a)-1}{\operatorname{sh}^{2}(\Delta a)\left(1-(\mu / \Delta)^{2}\right)} e^{\mu(b-a)} e^{-\Delta(b-a) \operatorname{cth}(\Delta a)}, \quad \mu \neq 0$,

$\mathbf{E} e^{-\lambda \gamma_{a b}^{0}}=\frac{1-e^{-(b-a) \sqrt{2 \lambda} \operatorname{cth}(a \sqrt{2 \lambda})}}{\operatorname{ch}(a \sqrt{2 \lambda})}+\frac{2 e^{-(b-a) \sqrt{2 \lambda} \operatorname{cth}(a \sqrt{2 \lambda})}}{\operatorname{ch}(a \sqrt{2 \lambda})+1} ;$

3. $\mathbf{P}\left(\gamma_{a b}=\tau_{a}\right)=1-m_{a} e^{-\frac{b-a}{\lambda_{a}}}, \quad \mathbf{P}\left(\gamma_{a b}=\sigma_{b}\right)=m_{a} e^{-\frac{b-a}{\lambda_{a}}}, \quad \mu \neq 0$,

$$
\mathbf{P}\left(\gamma_{a b}^{0}=\tau_{a}^{0}\right)=1-\frac{1}{2} e^{-\frac{b-a}{a}}, \quad \mathbf{P}\left(\gamma_{a b}^{0}=\sigma_{b}^{0}\right)=\frac{1}{2} e^{-\frac{b-a}{a}}
$$

4.

$\mathbf{E} \gamma_{a b}=\frac{e^{2 \mu a}-2 \mu a-1}{2 \mu^{2}}\left(1-m_{a} e^{-\frac{b-a}{\lambda_{a}}}\right), \quad \mu \neq 0, \quad \mathbf{E} \gamma_{a b}^{0}=a^{2}\left(1-\frac{1}{2} e^{-\frac{b-a}{a}}\right)$ 
Используя (2) и формулы обращения из [2; приложение 2], несложно найти плотность распределения момента $\gamma_{a b}$. Соответствующую формулу мы здесь не приводим ввиду ее громоздкости. Отметим также, что (3) уже было найдено ранее в [3] из других соображений.

2. Для $\mu \in \mathbb{R}$ и $a>0, c>0$ введем следующие моменты:

$$
\delta_{c}=\inf \left\{t \geqslant 0: B_{t}^{\mu}=c\right\}, \quad \zeta_{a c}=\tau_{a} \wedge \delta_{c}, \quad \rho_{a c}=\inf \left\{t \geqslant 0:\left|B_{t}\right|=a \text { или } L_{t}=c\right\},
$$

где $L_{t}$ - локальное время броуновского движения в нуле. Доказательство теоремы 1 основывается на следующих леммах.

Лемма 1. Пусть $B_{t}^{\mu}$ - броуновское движение со сносом $\mu$, моменты $\tau_{a}, \sigma_{a}, \gamma_{a b}$

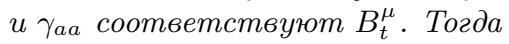

$$
\gamma_{a b}=\gamma_{a a}+\hat{\zeta}_{a, b-a} I_{\left\{\gamma_{a a}=\sigma_{a}\right\}}, \quad \tau_{a}=\gamma_{a a}+\hat{\tau}_{a} I_{\left\{\gamma_{a a}=\sigma_{a}\right\}},
$$

где моменты $\hat{\zeta}_{a, b-a}$ и $\hat{\tau}_{a}$ соответствуют независимой копии $\widehat{B}_{t}^{\mu}$ процесса $B_{t}^{\mu}$.

Лемма 2. Преобразование Лапласа тройки $\left(\rho_{a c}, L_{\rho_{a c}},\left|B_{\rho_{a c}}\right|\right)$ имеет вид

$$
\mathbf{E} e^{-\lambda \rho_{a c}+\beta L_{\rho_{a c}}+\gamma\left|B \rho_{a c}\right|}=\frac{e^{\gamma a}\left(1-e^{\beta c} e^{-c \sqrt{2 \lambda} \operatorname{cth}(a \sqrt{2 \lambda})}\right)}{\operatorname{ch}(a \sqrt{2 \lambda})-(\beta / \sqrt{2 \lambda}) \operatorname{sh}(a \sqrt{2 \lambda})}+e^{\beta c} e^{-c \sqrt{2 \lambda} \operatorname{cth}(a \sqrt{2 \lambda})}
$$

$\partial \Omega \Re \beta<\sqrt{2 \lambda} \operatorname{cth}(a \sqrt{2 \lambda})$.

3. Скажем коротко об основных идеях доказательства теоремы 1. Формула (2) является очевидным следствием (1). Представления (5) позволяют свести изучение $\gamma_{a b}$ к исследованию $\zeta_{a, b-a}$. Поэтому для доказательства (1), (3) и (4) достаточно найти $\mathbf{E} \exp \left\{-\lambda \zeta_{a, b-a}+\beta B_{\zeta_{a, b-a}}^{\mu}\right\}, \mathbf{P}\left(\zeta_{a, b-a}=\tau_{a}\right)$ и $\mathbf{E} \zeta_{a, b-a}$. Из теоремы Леви о совместном распределении $\sup _{s \leqslant t} B_{s}$ и $B_{t}$ и теоремы Гирсанова следует, что

$$
\mathbf{E} \exp \left\{-\lambda \zeta_{a, b-a}+\beta B_{\zeta_{a, b-a}}^{\mu}\right\}=\mathbf{E} \exp \left\{-\frac{\Delta^{2}}{2} \rho_{a, b-a}+\nu L_{\rho_{a, b-a}}-\nu\left|B_{\rho_{a, b-a}}\right|\right\} .
$$

Остается только подставить (6). Заметим, что

$$
\mathbf{P}\left(\zeta_{a, b-a}=\tau_{a}\right)=\mathbf{P}\left(\sup _{s \leqslant \tau_{a}} B_{s}^{\mu} \leqslant b-a\right)
$$

$\operatorname{aup}_{s \leqslant \tau_{a}} B_{s}^{\mu}$ имеет экспоненциальное распределение с параметром $1 / \lambda_{a}$ (см., например, [1]). Пользуясь строго марковским свойством броуновского движения, несложно получить

$$
\mathbf{E} \zeta_{a, b-a}=\mathbf{E} \tau_{a} \cdot \mathbf{P}\left(\zeta_{a, b-a}=\tau_{a}\right) .
$$

\section{Список литературы}

[1] А.Н. Ширяев, О мартингалъных методах в задачах о пересечении грании броуновским движением, МИАН, М., 2007. [2] А.Н. Бородин, П. Салминен, Справочник по броуновскому движению, Лань, СПб., 2000; пер. с англ.: А. N. Borodin, P. Salminen, Handbook of Brownian motion - facts and formulae, Probab. Appl., Birkhäuser Verlag, Basel, 1996. [3] O. Hadjiliadis, J. Vecer, Quant. Finance, 6:5 (2006), 403-409.

\section{А. А. Муравлёв (А. А. Muravlev)}

Московский государственный университет им. М. В. Ломоносова

E-mail: almurav@gmail.com
Представлено А. В. Булинским Принято редколлегией 13.10 .2008 